\section{Die katalytische Aktivierung von Wasserstoff an mit Übergangsmetallionen dotiertem Korund}

\section{H. Schaefer und E. Büchler}

Fritz-Haber-Institut der Max-Planck-Gesellschaft, Berlin-Dahlem

(Z. Naturforsch. 23 a, 1685-1686 [1968] ; eingeg. am 30. August 1968)

Übergangsmetallionen, in hoher Verdünnung in a- $\mathrm{Al}_{2} \mathrm{O}_{3}$ (Korund) isovalent eingebaut, sind auf Grund der elektrischen und magnetischen Eigenschaften des Korundes in dieser katalytisch wenig aktiven Matrix praktisch als isoliert zu betrachten. Eventuelle katalytische Eigenschaften solcher festen Lösungen sollten sich daher in guter Näherung als lokalisierte Wechselwirkungen zwischen Reaktanten und Übergangsmetallionen in der Koordinationssphäre ihrer nächsten Sauerstoffnachbarn beschreiben lassen. Wir berichteten kürzlich $^{1}$ (siehe dort auch Hinweise auf die einschlägige Literatur), daß die $\mathrm{C}_{2} \mathrm{H}_{4}$-Hydrierung an $\mathrm{Cr}^{3+}$, aber nicht an $\mathrm{Ti}^{3^{+}}$-dotiertem Korund im Vergleich zu reinem Korund beschleunigt abläuft. Inzwischen ergänzend durchgeführte Untersuchungen zeigten außerdem, daß in Korund eingebaute $\mathrm{V}^{3^{+}}$-Ionen ebenfalls beschleuni- gend wirken ${ }^{2}$. Im folgenden teilen wir die Ergebnisse einiger Versuche mit, in denen wir die Aktivierung eines der Reaktionsteilnehmer, nämlich des Wasserstoffs, studierten.

Wir untersuchten den $\mathrm{H}_{2}-\mathrm{D}_{2}$-Austausch bei Normaldruck in einer Strömungsapparatur an mit $\mathrm{Ti}^{3^{+}}, \mathrm{V}^{3^{+}}$ und $\mathrm{Cr}^{3^{+}}$dotiertem Korund. Die Darstellung der Kontakte war früher ${ }^{1}$ beschrieben worden. Vor der Messung wurden die Kontakte durch etwa 12-stündiges Ausheizen auf $650{ }^{\circ} \mathrm{C}$ in strömendem $\mathrm{He}$ oder $\mathrm{H}_{2}$ aktiviert. Auf sorgfältigen Ausschluß von $\mathrm{H}_{2} \mathrm{O}$ und $\mathrm{O}_{2}$ wurde geachtet.

Der Einbau von $\mathrm{Cr}^{3^{+}}$-Ionen erhöht deutlich die katalytische Aktivität des reinen Korundes für den $\mathrm{H}_{2}-\mathrm{D}_{2}$ Austausch (Abb. 1). Nach Heliumaktivierung (Kurve 1) nimmt mit steigender Temperatur die Aktivität bis ungefähr $200{ }^{\circ} \mathrm{C} \mathrm{zu}$, um bei noch höheren Temperaturen wieder abzufallen. Die absolute Größe der gemessenen Aktivitäten ist hierbei davon abhängig, wie schnell die Messungen durchgeführt werden. Längerer Aufenthalt in $\mathrm{H}_{2}$ oberhalb $200{ }^{\circ} \mathrm{C}$ während der Messung und vor allem Wasserstoffbehandlung bei $650{ }^{\circ} \mathrm{C}$ (Kurve 2) vergiften die Kontakte. Erneute Aktivierung in $\mathrm{He}$ gibt dem Katalysator, der zwischendurch nicht mit Sauer-

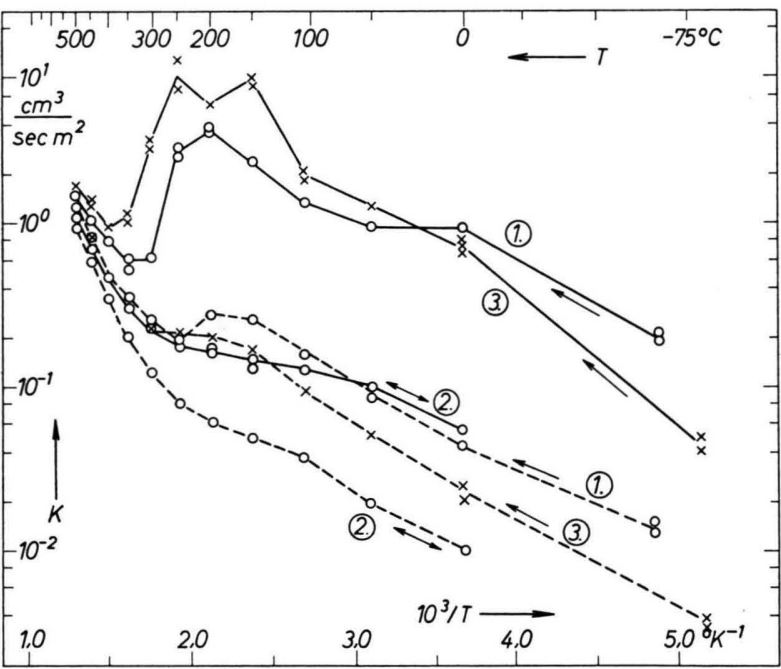

Abb. 1. Spezifische Geschwindigkeitskonstanten $(K)$ erster Ordnung für den $\mathrm{H}_{2}-\mathrm{D}_{2}$-Austausch an undotiertem (gestrichelt) und mit 1,2 AtomProz. $\mathrm{Cr}^{3^{+}}$dotiertem Korund (ausgezogen). Die Pfeile geben die Richtung der Temperaturänderung während der Messung an. 1) und 3) in $\mathrm{He}$ bei $650^{\circ}$ aktiviert und abgekühlt, 2) in $\mathrm{H}_{2}$ bei $650^{\circ}$ aktiviert und abgekühlt.

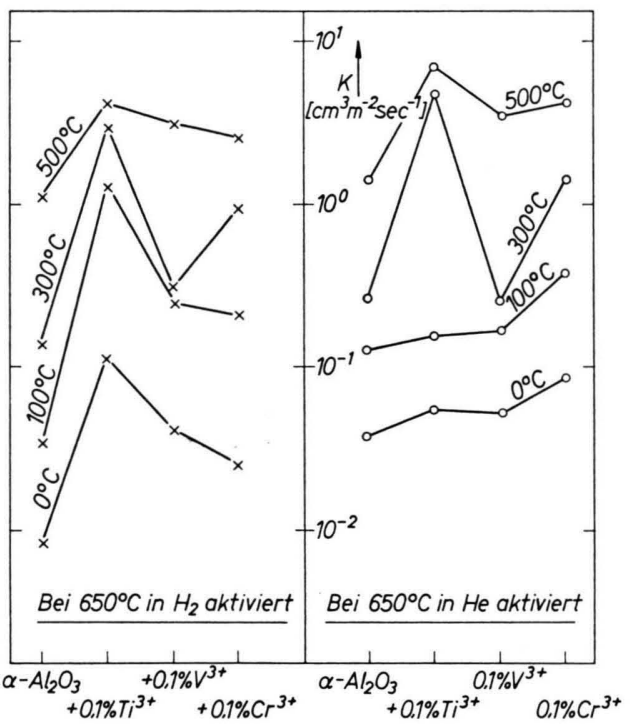

Abb. 2. Spezifische Geschwindigkeitskonstanten $(K)$ erster Ordnung für den $\mathrm{H}_{2}-\mathrm{D}_{2}$ Austausch an undotiertem und mit je 0,1 Atom-Proz. $\mathrm{Ti}^{3+}, \mathrm{V}^{3+}$ und $\mathrm{Cr}^{3^{+}}$dotiertem Korund. Die an den Kurven vermerkten Temperaturen geben die Meßtemperaturen
1 H.Schaefer u. E. Büchler, Z. Naturforsch. 22 a, 2117 [1967].

2 Nach Aktivierung in strömendem $\mathrm{He}$ bei $650^{\circ} \mathrm{C}$ (früher $550^{\circ} \mathrm{C}$ ) für 12 Stunden wird bei Anwendung der mikrokatalytischen Pulstechnik in der Art, wie früher ${ }^{1}$ beschrieben, bei $20^{\circ} \mathrm{C}$ beobachtet (eingesetzte Oberfläche je 0,228 $\mathrm{m}^{2}, \mathrm{H}_{2}$-Trägergas $1,14 \mathrm{ml} / \mathrm{sec}$ ), daß an undotiertem und mit 0,1 Atom-Prozent $\mathrm{Ti}^{3+}$ dotiertem Korund höchstens 3,5\% des eingesetzten Äthylens umgesetzt werden. Dagegen werden an Korund, der mit 0,1 Atom-Proz. $\mathrm{Cr}^{3+}$ oder $\mathrm{V}^{3+}$ dotiert war, ca. 44 bzw. $57 \% \mathrm{C}_{2} \mathrm{H}_{6}$ gebildet. Ausheizen bei $650^{\circ}$ und Abkühlen in strömendem $\mathrm{H}_{2}$ verringert die Aktivität der $\mathrm{Cr}^{3+}$-dotierten (ca. $7 \% \mathrm{C}_{2} \mathrm{H}_{6}$ ) und erhöht die Aktivität der $\mathrm{V}^{3+}$-dotierten Probe (ca. $93 \% \mathrm{C}_{2} \mathrm{H}_{6}$ ). 
stoff in Berührung gekommen ist, wieder die alte Aktivität (Kurve 3). Die gleiche Wirkung wie die Heliumbehandlung hat auch Erhitzen im Hochvakuum. Dieses Verhalten deutet darauf hin, daß der schnelle $\mathrm{H}_{2}-\mathrm{D}_{2}$ Austausch bei tiefen und mittleren Temperaturen nach He-Aktivierung von einer sehr reaktiven Form der $\mathrm{H}_{2}$ Adsorption bewirkt wird, die durch eine oberhalb $200{ }^{\circ} \mathrm{C}$ einsetzende, wenig reaktionsfähige und fest gebundene zweite $\mathrm{H}_{2}$-Adsorption gehemmt werden kann. Dazu im Gegensatz wirkt $\mathrm{H}_{2}$-Behandlung bei $650{ }^{\circ} \mathrm{C}$ an der $\mathrm{Ti}^{3^{+}}$-dotierten Probe (unterhalb $100^{\circ}$ ) stark beschleunigend (Abb. 2; mangelnde Reproduzierbarkeit des Aktivierungsprozesses wurde nach Vergleich mit der Aktivität eines jeweils gleichbehandelten und parallel vermessenen undotierten Korundes korrigiert). Dementsprechend fällt der Aktivitätsvergleich der verschiedenen Dotierungen nach $\mathrm{H}_{2}$ - und He-Aktivierung unterschiedlich aus (Abb. 2). Nach $\mathrm{H}_{2}$-Aktivierung wird im ganzen untersuchten Temperaturintervall die höchste Aktivität an der $\mathrm{Ti}^{3^{+}}$-dotierten Probe beobachtet. Nach He-Aktivierung ist dagegen bei mittleren und tiefen Temperaturen, also solange die Hochtemperaturadsorption des Wasserstoffs nicht einsetzt, der $\mathrm{Cr}^{3^{+}}$. dotierte Korund besonders aktiv.

Unsere Versuche zeigen also, daß auch beim $\mathrm{H}_{2}-\mathrm{D}_{2}$ Austausch die Elektronenkonfiguration der in guter Näherung in der Koordinationssphäre ihrer nächsten
Sauerstoffnachbarn isoliert zu betrachtenden Übergangsmetallionen einen großen Einfluß auf die katalytische Aktivität ausübt. Hierbei kann im einzelnen die Aktivität noch in charakteristischer Weise durch eine erst bei höheren Temperaturen auftretende $\mathrm{H}_{2}$-Adsorntion modifiziert werden. Die beim $\mathrm{H}_{2}-\mathrm{D}_{2}$-Austausch beobachtete Aktivitätsfolge geht nicht derjenigen parallel, die bei der Äthylenhydrierung an $\mathrm{Ti}^{3^{+}}$-, $\mathrm{V}^{3^{+}}$- und $\mathrm{Cr}^{3^{+}}$-dotiertem Korund beobachtet wurde. Die von uns für den Austausch am $\mathrm{Cr}^{3^{+}}$-dotierten Korund mitgeteilten Ergebnisse stehen in einem gewissen Gegensatz zu Beobachtungen von $\mathrm{S}_{\mathrm{ELwood}}{ }^{3}$, der fand, daß in diesem System der $\mathrm{H}_{2}-\mathrm{D}_{2}$-Austausch durch $\mathrm{Cr}^{3+}$-Ionen nicht beschleunigt wird. Die Ursache für diese Diskrepanz ist nicht geklärt. Sie ist aber vermutlich in den von SELwoop und uns unterschiedlich gewählten Aktivierungsbedingungen für die Kontakte zu suchen.

Für die Förderung und freundliche Unterstützung dieser Arbeit danken wir Herrn Prof. Dr. R. Brill besonders herzlich. Den Firmen Siemens, München, und Union Carbide, Linde Division, Speedway Lab., Indianapolis, Ind., sind wir für die kostenlose Überlassung von undotierten und dotierten Korund-Verneuil-Kristallen sehr zu Dank verpflichtet.

3 P. W. Selwood, J. Am. Chem. Soc. 87, 1804 [1965] ; 88, 2676 [1966] ; Fourth Internat. Congr. on Catalysis, Moscow 1968, preprint 66. 